\title{
SOME RESULTS ON COHERENT RINGS
}

\author{
MORTON E. HARRIS ${ }^{1}$
}

1. Introduction. In [1, pp. 62-63, Exercises 11, 12] Bourbaki introduces a generalization of a left (resp. right) noetherian ring called a left (resp. right) coherent ring and states some properties and gives some examples of coherent rings. The results on coherent rings stated in $[1$, p. 63, Exercises 12a, g] first appeared in [3] which also gives other applications of coherent rings.

In $\S 2$ of this note we show, among other results, that if a ring $A$ is left (resp. right) coherent so is $A_{n}$, the $n \times n$ matrix ring with coefficients in $A$, that if $A$ is a commutative coherent ring then so is $A_{S}$, the ring of fractions of $A$ with respect to any multiplicative system $S$ in $A$, and that if $A$ is a coherent integral domain then so is any finite integral extension of $A$. In $\S 3$ we give examples of rings all of which are right coherent but not right noetherian and which have various properties on the left and we deduce two corollaries.

Throughout this note all rings will be assumed to contain an identity, all ring homomorphisms will be assumed to map identities into identities and all modules will be assumed to be unitary. If the underlying ring is noncommutative all definitions and results will be given for "one side," the "other side" result will be immediate by interchanging right and left. For the ring constructions of $\$ 3$, the "other side" result is obtained by taking anti-isomorphic rings.

Let $A$ be a ring and let $M$ be a left $A$-module then $M$ is finitely presented if there exists an exact sequence $F_{1} \rightarrow F_{0} \rightarrow M \rightarrow 0$ where $F_{1}$, $F_{0}$ are finitely generated free left $A$-modules (i.e. there exists a finitely generated free left $A$-module $F_{0}$ and a surjective $A$-homomorphism $u: F_{0} \rightarrow M$ whose kernel is a finitely generated $A$-module). Obviously every finitely presented left $A$-module is finitely generated and every finitely generated left $A$-module is finitely presented if $A$ is left noetherian. A useful result in this connection is given in $[1, \mathrm{p} .37]$ :

Lemma. Let $A$ be any ring and let $M$ be a finitely presented left $A$ module. Then if $0 \rightarrow F \rightarrow G \rightarrow M \rightarrow 0$ is an exact sequence of left $A$-modules where $G$ is finitely generated over $A$ then so is $F$.

As defined in $[1$, p. 62, Exercise 11] a left $A$-module $M$ is pseudo-

Received by the editors February 6, 1965 .

1 The research in this paper was supported in part by a Tufts University Faculty Summer Research Grant. The author wishes to express his gratitude to Professors George Leger and Pierre Samuel for their suggestions and advice. 
coherent if every finitely generated submodule of $M$ is finitely presented and $M$ is coherent if $M$ is both pseudo-coherent and finitely generated. Finally, as defined in [1, p. 63, Exercise 12] a ring $A$ is left coherent if $A$ as a left $A$-module is coherent.

2. Ring extensions. Among other results in this section we show that if $A$ is a left coherent ring then so is $A_{n}$, if $A$ is a commutative coherent ring then so is $A_{S}$ where $S$ is any multiplicative system in $A$ and that if $A$ is a coherent integral domain then so is any finite integral extension of $A$.

Theorem 1. Let $\phi: A \rightarrow B$ be a ring homomorphism such that $B$ as a left $A$-module by means of $\phi$ is finitely generated. If $M$ is a left $B$-module which when viewed as a left $A$-module (by means of $\phi$ ) is finitely presented then $M$ is a finitely presented left B-module.

Proof. Clearly $M$ is a finitely generated left $B$-module, say $M=\sum_{i=1}^{n} B m_{i}$. This gives rise to an exact sequence $0 \rightarrow K \rightarrow B^{n} \rightarrow M$ $\rightarrow 0$ of left $B$-modules where $\pi: B^{n} \rightarrow M$ is defined by: $\pi\left(\left(b_{i}\right)_{1 \leq i \leq n}\right)$ $=\sum_{i=1}^{n} b_{i} m_{i}$ and where $K$ is the kernel of $\pi$. Since $B^{n}$ is a finitely generated $A$-module and $M$ is a finitely presented $A$-module we conclude from the lemma of $\S 1$ that $K$ is a finitely generated $A$-module and thus a finitely generated $B$-module.

CoROllary 1.1. Let $\phi: A \rightarrow B$ be a ring homomorphism such that $B$ is a finitely generated left $A$-module by means of $\phi$. If $M$ is a left $B$ module which is pseudo-coherent (resp. coherent) as a left $A$-module then $M$ is a pseudo-coherent (resp. coherent) left B-module.

Proof. Observe that every finitely generated $B$-submodule of $M$ is a finitely generated $A$-submodule of $M$ and apply Theorem 1.

CoRollary 1.2. Let $\phi: A \rightarrow B$ be a ring homomorphism such that $B$ is a finitely presented left $A$-module by means of $\phi$. If $A$ is left coherent then so is $B$.

Proof. If $A$ is left coherent then every finitely presented left $A$ module is coherent [1, p. 63, Exercise 12a]. Apply Corollary 1.1 with $M=B$.

CoRollary 1.3. If $A$ is a left coherent ring then $A_{n}$, the ring of $n \times n$ matrices with coefficients in $A$ is also left coherent.

Proof. Trivial, use the canonical injection $A \rightarrow A_{n}$.

Corollary 1.4. Let $A$ be a coherent integral domain, let $K$ be a field containing $A$ and let $x \in K$ be integral over $A$ (i.e. $x$ satisfies a monic 
polynomial with coefficients in $A)$. Then $A[x]$ is a coherent ring.

Proof. Since $A[x]$ is a finitely generated torsion free $A$-module it is isomorphic to a submodule of a finitely generated free $A$-module $F$ by $[2$, p. 131 , Proposition 2.4]. Since $F$ is a pseudo-coherent $A$-module $[1$, p. 62 , Exercise $11 \mathrm{c}]$ we conclude that $A[x]$ is a finitely presented $A$-module and hence that $A[x]$ is coherent by Corollary 1.3.

COROLlary 1.5. Every finite integral extension of a coherent integral domain is coherent.

Corollary 1.6. Let $A$ be a coherent integral domain and $K$ a field containing $A$. Assume that whenever $A_{\alpha}, A_{\beta}$ are two finite integral extensions of $A$ in $K$ such that $A_{\beta}$ is a flat $A_{\alpha}$ module when $A_{\alpha} \subseteq A_{\beta}$ then the integral closure of $A$ in $K$ is coherent.

Proof. Apply $[1$, p. 63, Exercise 12e] to the directed system composed of the finite integral extensions of $A$ in $K$ and their injections.

TheOREM 2. Let $\phi: A \rightarrow B$ be a ring homomorphism such that $B$ is a faithfully flat right $A$-module by means of $\phi$. Then if $E$ is a left $A$ module such that $B \otimes_{A} E$ is a pseudo-coherent (resp. coherent) left $B$ module then $E$ is a pseudo-coherent (resp. coherent) left A-module.

Proof. By [1, p. 51, Proposition 9], $\phi$ is an injection so that $A$ may be viewed as a subring of $B$. Let $E^{\prime}$ be a finitely generated $A$-submodule of $E$, hence $B \otimes_{A} E^{\prime}$ is a finitely presented left $B$-module. Thus by $\left[1\right.$, p. 52 , Proposition 11] we conclude that $E^{\prime}$ is a finitely presented $A$-module and hence $E$ is pseudo-coherent. From the same proposition it follows that if $B \otimes_{A} E$ is a finitely generated $B$-module then $E$ is a finitely generated $A$-module.

Corollary 2.1. If $\phi: A \rightarrow B$ is a ring homomorphism where $B$ as a right $A$-module by means of $\phi$ is faithfully flat and if $B$ is left coherent then so is $A$.

Corollary 2.2. If $A$ is a ring such that $A_{n}$ is left coherent for some positive integer $n$ then so is $A_{m}$ for all positive integers $m$.

For the remainder of this section, $A$ will denote a commutative ring, $S$ a multiplicative system in $A, A_{S}$ the ring of fractions of $A$ with respect to $S$ and if $M$ is an $A$-module then $M_{S}$ will denote the module of fractions of $M$ with respect to $S$ which becomes an $A_{S}$ module.

Theorem 3. If $M$ is a pseudo-coherent (resp. coherent) $A$-module then $M_{S}$ is a pseudo-coherent (resp. coherent) $A_{S}$-module. 
Proof. Clearly if $M$ is a finitely generated $A$-module then $M_{S}$ is a

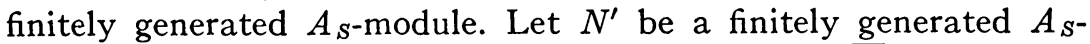
submodule of $M_{S}$, hence we may assume that $N^{\prime}=\sum_{i=1}^{n} A_{S}\left(m_{i} / 1\right)$ where $m_{i} \in M$ for $1 \leqq i \leqq n$. Set $N=\sum_{i=1}^{n} A m_{i}$, then $N$ is a finitely presented $A$-module and hence $N_{S}$ is a finitely presented $A_{S}$ module. But $N_{S}$ is isomorphic over $A_{S}$ to $N^{\prime}$ since $A_{S}$ is a flat $A$-module and so $N^{\prime}$ is a finitely presented $A_{S}$-module.

Corollary 3.1. If $A$ is a commutative coherent ring then so is $A_{s}$ for any multiplicative system $S$ in $A$.

3. Examples. Here we construct rings which are right coherent but not right noetherian and which on the left can be noetherian, not noetherian, or not coherent and we deduce two corollaries.

Left and right may be interchanged in all results in this section by taking anti-isomorphic rings.

Let $A$ be a right noetherian ring and after the example on $[2$, p. 16] let $R=A[X, Y]$ be the noncommutative polynomial ring in two indeterminates $X, Y$ with coefficients in $A$ where $Y Y=Y X=0$. Thus $R=A[X]+A[X] Y$ is not right noetherian since $\left\{X^{n} Y \mid 1 \leqq n\right\}$ generates a nonfinitely generated right ideal of $R$. We proceed to show that $R$ is right coherent.

Let $\left\{f_{i}(X)+g_{i}(X) Y \mid 1 \leqq i \leqq s\right\}$ be $s$ elements of $R$ which generate a right ideal $I \neq(0)$ of $R$ and let $\pi: R^{s} \rightarrow I$ be defined by:

$$
\pi\left(\left(p_{i}(X)+m_{i}(X) Y\right)_{1 \leqq i \leqq s}\right)=\sum_{i=1}^{s}\left(f_{i}(X)+g_{i}(X) Y\right)\left(p_{i}(X)+m_{i}(X) Y\right) .
$$

Clearly $\pi$ is a right $R$-module homomorphism. It must be shown that $\operatorname{Ker}(\pi)$ is a finitely generated $R$-submodule of $R^{s}$; it suffices to assume that $\operatorname{Ker}(\pi) \neq(0)$.

Now $\pi\left(\left(p_{i}(X)+m_{i}(X) Y\right)_{1 \leqq i \leqq s}\right)=0$ if and only if :

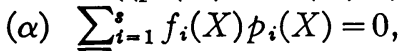

(ß) $\sum_{i=1}^{s}\left(f_{i}(X) m_{i}(X)+g_{i}(X) p_{i}(0)\right)=0$.

Let $\mathfrak{A}=\sum_{i=1}^{s} f_{i}(X) A[X]$ and turn $A[X] / \mathfrak{A}$ into a right $A[X]$ module by defining $(f(X)+\mathfrak{X}) g(X)=f(X) g(0)+\mathfrak{X}$. Define $\theta:(A[X])^{\circ}$ $\rightarrow A[X] \oplus A[X] / \mathfrak{A}$ by:

$$
\begin{aligned}
\theta\left(\left(p_{i}(X)\right)_{1 \leqq i \leqq s}\right) & =\left(\sum_{i=1}^{8} f_{i}(X) p_{i}(X), \sum_{i=1}^{8}\left(g_{i}(X)+\mathfrak{A}\right) p_{i}(X)\right) \\
& =\left(\sum_{i=1}^{8} f_{i}(X) p_{i}(X), \sum_{i=1}^{8} g_{i}(X) p_{i}(0)+\mathfrak{P}\right) .
\end{aligned}
$$

Clearly $\theta$ is a homomorphism of right $A[X]$ modules and $\operatorname{Ker}(\theta)$ is a 
finitely generated right $A[X]$ module since $A[X]$ is right noetherian. If $\left(p_{i}(X)+m_{i}(X) Y\right)_{1 \leq i \leq s} \in \operatorname{Ker}(\pi)$ then $\left(p_{i}(X)\right)_{1 \leqq i \leqq s} \in \operatorname{Ker}(\theta)$ and conversely if $\left(p_{i}(X)\right)_{1 \leq i \leq s} \in \operatorname{Ker}(\theta)$ then there exists an element $\left(m_{i}(X)\right)_{1 \leqq i \leqq s} \in(A[X])^{s}$ such that $\left(p_{i}(X)+m_{i}(X) Y\right)_{1 \leqq i \leqq s} \in \operatorname{Ker}(\pi)$. Moreover if $\left(m_{i}(X) Y\right)_{1 \leqq i \leqq s} \in \operatorname{Ker}(\pi)$ then $\sum_{i=1}^{s} f_{i}(X) m_{i}(X)=0$ and hence $\left(m_{i}(X) X\right)_{1 \leq i \leq s} \in \operatorname{Ker}(\theta)$. Hence $\operatorname{Ker}(\pi) \neq(0)$ implies $\operatorname{Ker}(\theta)$ $\neq(0)$.

Let $\alpha_{j}=\left(p_{i}^{j}(X)\right)_{1 \leqq i \leq s}$ for $1 \leqq j \leqq r$ generate $\operatorname{Ker}(\theta)$ over $A[X]$ and for each $\alpha_{j}$ choose $\left(m_{i}^{j}(X)\right)_{1 \leqq i \leqq s} \in(A[X])^{s}$ such that $\alpha_{j}^{*}=\left(p_{i}^{j}(X)\right.$ $\left.+m_{i}^{j}(X) Y\right)_{1 \leqq i \leqq s} \in \operatorname{Ker}(\pi)$ for $1 \leqq j \leqq r$. Let $\mathscr{B}=\sum_{j=1}^{r} \alpha_{j}^{*} R \subseteq \operatorname{Ker}(\pi)$. If $\left(p_{i}(X)+m_{i}(X) Y\right)_{1 \leq i \leq s} \in \operatorname{Ker}(\pi)$ then $\left(p_{i}(X)\right)_{1 \leqq i \leqq s} \in \operatorname{Ker}(\theta)$ so that there exists an element $\left(h_{i}(X)\right)_{1 \leqq i \leqq r} \in(A[X])^{r}$ such that $\left(p_{i}(X)\right)_{1 \leqq i \leqq s}$ $=\sum_{j=1}^{r} \alpha_{j} h_{j}(X)$, whence $\left(p_{i}(X)+m_{i}(X) Y\right)_{1 \leqq i \leqq s}-\sum_{j=1}^{r} \alpha_{j}^{*} h_{j}(X)$ is of the form:

$$
\left(m_{i}^{*}(X) Y\right)_{1 \leq i \leq 8}
$$

To conclude that $R$ is right coherent we will show that there exists a finitely generated $R$-submodule $\mathfrak{E}$ of $\operatorname{Ker}(\pi)$ such that $\mathfrak{B}+\mathfrak{E}=\operatorname{Ker}(\pi)$.

Let

$$
M=\left\{\left(m_{i}(X)\right)_{1 \leq i \leq s} \in(A[X])^{s} \mid\left(m_{i}(X) Y\right)_{1 \leq i \leq s} \in \operatorname{Ker}(\pi)\right\}
$$

and

$$
M^{*}=\left\{\left(m_{i}(X)\right)_{1 \leq i \leq s} \in A([X])^{s} \mid\left(m_{i}(X) Y\right)_{1 \leq i \leq s} \in \mathfrak{B}\right\}
$$

and define $\rho: M \rightarrow A^{s}$ by $\rho\left(\left(m_{i}(X)\right)_{1 \leqq i \leqq s}\right)=\left(m_{i}(0)\right)_{1 \leqq i \leqq s}$. Clearly $\rho$ is a homomorphism of right $A$-modules. We claim that $\operatorname{Ker}(\rho) \subseteq M^{*}$ for if $\left(m_{i}(X)\right)_{1 \leqq i \leqq s} \in \operatorname{Ker}(\rho)$ then $\left(m_{i}(X)\right)_{1 \leqq i \leqq s} \in \operatorname{Ker}(\pi)$ and as before there exists an element $\left(p_{i}^{\prime}(X)+m_{i}^{\prime}(X) Y\right)_{1 \leq i \leq s} \in \mathfrak{B}$ such that $\left(m_{i}(X)\right)_{1 \leq i \leq s}$ $-\left(p_{i}^{\prime}(X)+m_{i}^{\prime}(X) Y\right)_{1 \leqq i \leqq s}$ is of form $\left(^{*}\right)$. Therefore $\left(m_{i}(X) Y\right)_{1 \leq i \leq s}$ $=\left(p_{i}^{\prime}(X) Y\right)_{1 \leqq i \leqq s} \in \mathcal{B}$ or $\left(m_{i}(X)\right)_{1 \leqq i \leqq s} \in M^{*}$.

Since $A$ is right noetherian we may choose $\beta_{j}=\left(n_{i}^{j}(X)\right)_{1 \leq i \leq s} \in M$, $1 \leqq j \leqq t$, such that $\rho(M)=\sum_{j=1}^{t} \rho\left(\beta_{j}\right) A$. Set $\beta_{j}^{*}=\left(n_{i}^{j}(X) Y\right)_{1 \leq i \leq s}$ $\in \operatorname{Ker}(\pi)$ for $1 \leqq j \leqq t$ and let $\left(m_{i}(X) Y\right)_{1 \leq i \unlhd s} \in \operatorname{Ker}(\pi)$. Then there exists an element $\left(a_{j}\right)_{1 \leq j \leq t} \in A^{t}$ such that

$$
\left(m_{i}(X)\right)_{1 \leq i \leq s}-\sum_{j=1}^{t} \beta_{j} a_{j} \in \operatorname{Ker}(\rho) \subseteq M^{*},
$$

whence $\left(m_{i}(X) Y\right)_{1 \leqq i \leqq s}-\sum_{j=1}^{t} \beta_{j}^{*} a_{j} \in \mathfrak{B}$. Thus if $\mathfrak{C}=\sum_{j=1}^{t} \beta_{j}^{*} R$ then $\mathfrak{C} \subseteq \operatorname{Ker}(\pi)$ and all elements of $\operatorname{Ker}(\pi)$ of form $\left(^{*}\right)$ are contained in $\mathfrak{B}+\mathfrak{C}$. Hence $\operatorname{Ker}(\pi)=\mathfrak{B}+\mathfrak{C}$. 
Theorem 4. If $A$ is a right noetherian ring then $R=A[X, Y]$, the noncommutative polynomial ring in $X, Y$ with coefficients in $A$ where $Y Y=Y X=0$ is right coherent but not right notherian and

(a) if $A$ is left noetherian so is $R$,

(b) if $A$ is not left noetherian neihter is $R$,

(c) if $A$ is not left coherent neither is $R$. (In $[1, p .63$, Exercise 11b] an example is given of a right noetherian ring which is not left coherent).

Proof. (a). If $A$ is left noetherian, then $A[X]$ is left noetherian and so is the finitely generated left $A[X]$ module $R=A[X]+A[X] Y$.

(b), (c). Let $\mathfrak{D}=R X R+R Y R$ then $R / \mathfrak{D}$ is isomorphic to $A$ so that if $R$ is left noetherian so is $A$. Moreover $\mathfrak{D}=R X+R Y$ so that if $R$ is left coherent so is $A$ by a remark on [3, pp. 463-464].

COROLlary 4.1. There exists a finitely generated ring which is right coherent but not right noetherian.

Proof. Take $A$ to be a finite ring and generate $R$ as above.

One might conjecture that coherent rings are obtained as direct limits of noetherian rings but:

COROLlaRy 4.2. There exists a right coherent ring which is not a direct limit of right noetherian rings.

Proof. Take $R$ as in Corollary 4.1 and suppose that $R$ is the direct limit of a directed system of right noetherian rings $\left\{A_{\alpha}\right\}$. Since $R$ is a finitely generated ring it is a homomorphic image of some $A_{\alpha}$ and hence $R$ is right noetherian-contradiction.

\section{REFERENCES}

1. N. Bourbaki, Algèbre commutative, Chapitres 1-2, Hermann, Paris, 1961.

2. H. Cartan and S. Eilenberg, Homological algebra, Princeton Univ. Press, Princeton, N. J., 1956.

3. S. U. Chase, Direct products of modules, Trans. Amer. Math. Soc. 97 (1960), $457-473$.

TUFTS UNIVERSITY 